\title{
Liver Hydatid Cyst Complicated by Biliary and Colonic Fistula Diagnosed after Intra-Operative Cholangiography
}

\author{
Karim Nacef, Mohamed Ali Chaouch, Mohamed Ben Khalifa, Asma Chaouch, \\ Mossab Ghannouchi, Mohamed Maatouk, and Moez Boudokhane
}

\begin{abstract}
Background: Hydatid disease is a worldwide zoonosis and is localized in the liver in most cases. Biliary rupture and infection are the most common complications of hydatid liver. Simultaneous biliary and colonic fistula is uncommon. Clinical diagnoses is difficult, however, ultrasound echography and abdominal computed tomography (CT) scan can detect these complications.

Case Presentation: Our case was diagnosed by a peri-operative trans-cystic cholangiography showing simultaneous opacification of liver hydatid cyst and the right colonic angle. The decision was to disconnect the kystocolonic fistula and create colonic stoma on the colic defect followed by drainage of the residual cavity. The course was marked by the death of the patient after four days caused by respiratory and renal failure.

Conclusion: This abnormal communication is always secondary to hydatid-cyst infection caused by hydatid cystbiliary fistula. Surgical treatment is sometimes difficult and prognosis depends on the gastrointestinal organ affected.
\end{abstract}

Keywords: colonic; fistula; hytatid cyst; infection

$\mathbf{H}$ EPATIC HYDATIDOSIS is an anthropozoonosis caused by Echinococcus granulosus. Diagnosis is often made when the cyst is complicated because of its clinical latency [1]. Rupture in the digestive tract is rare, occurring in $0.15 \%$ to $0.29 \%$ of cases [2,3]. Generally ultrasound and computed tomography (CT) scan can detect this complication. In our case it was diagnosed by an intra-operative cholangiography. The goal of this work is to specify the uncommon radiologic findings of hydatid cyst-bilio-colonic fistula diagnosed by a trans-cystic intra-operative cholangiography showing simultaneous opacification of the biliary tract, liver hydatid cyst, and right colonic angle.

\section{Case Presentation}

An 81-year-old female with chronic renal failure, who consulted for acute right hypochondriac pain presented with fever (temperature, $38.5^{\circ} \mathrm{C}$ ). Abdominal examination revealed a defect in the right upper quadrant. Biologic examinations revealed an inflammatory biologic syndrome with high white blood cell count $(15,000$ cells/mcL) and elevated
C-reactive protein $(31 \mathrm{mg} / \mathrm{L})$ without hepatic cytolysis or cholestasis. Ultrasound and abdominal CT scan found lithiasis cholecystitis, calcified and infected hydatid cyst in segment IV with probable hydatid cyst-colonic fistula (Fig. 1). Through a right sub-costal incision, the gallbladder was found to be gangrenous and contained fecal matter without cholecysto-digestive fistula. A cholecystectomy with cholangiography was performed. A normal common bile duct was shown, associated with simultaneous opacification of a hydatid cyst cavity and the right colonic angle (Fig. 2). The diagnosis of a hydatid cyst, biliary and colonic communication was made. We had to disconnect the hydatid cyst-biliary fistula using direct suture and create lateral colonic stoma on the colonic right angle defect followed by drainage of the residual cavity. However, the patient died four days later because of renal and respiratory failure.

\section{Discussion}

Hepatic is the most common location of a hydatid cyst. Its evolution can be marked by serious complications, the most

General Department of Surgery, Taher Sfar Hospital, Mahdia, Tunisia.

(C) Karim Nacef et al. 2017; Published by Mary Ann Liebert, Inc. This Open Access article is distributed under the terms of the Creative Commons License (http://creativecommons.org/licenses/by/4.0), which permits unrestricted use, distribution, and reproduction in any medium, provided the original work is properly credited. 


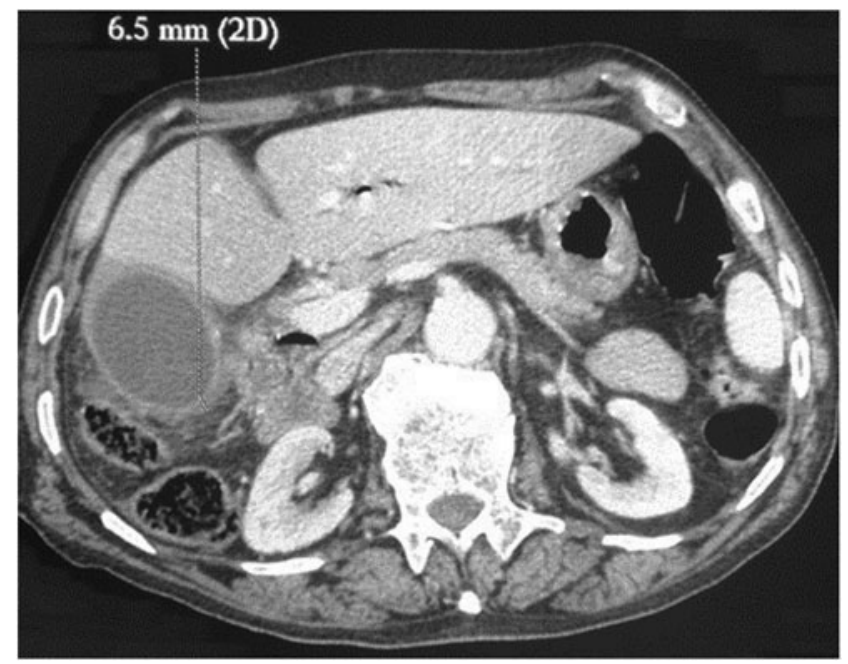

FIG. 1. Abdominal computed tomography (CT) scan with lithiasis cholecystitis and doubt on hydatid cyst-colonic fistula.

common of which is the communication with biliary tract. Rupture in the gastrointestinal tract is rare: $0.29 \%$ according to Kourias et al. [2] and $0.15 \%$ according to Zaouche et al. [3]. Few cases have been reported in the literature [4-6]. Hydatid cyst involves the stomach and duodenum fistula [7] but can also involve abdominal esophagus, stomach, duodenum, colon, or even small bowel. This complication occurs after several stages. Initially, the hydatid cyst communicates with bile ducts, bacterial infection occurs [8], mechanical factor by compression results from increased cyst size gen-

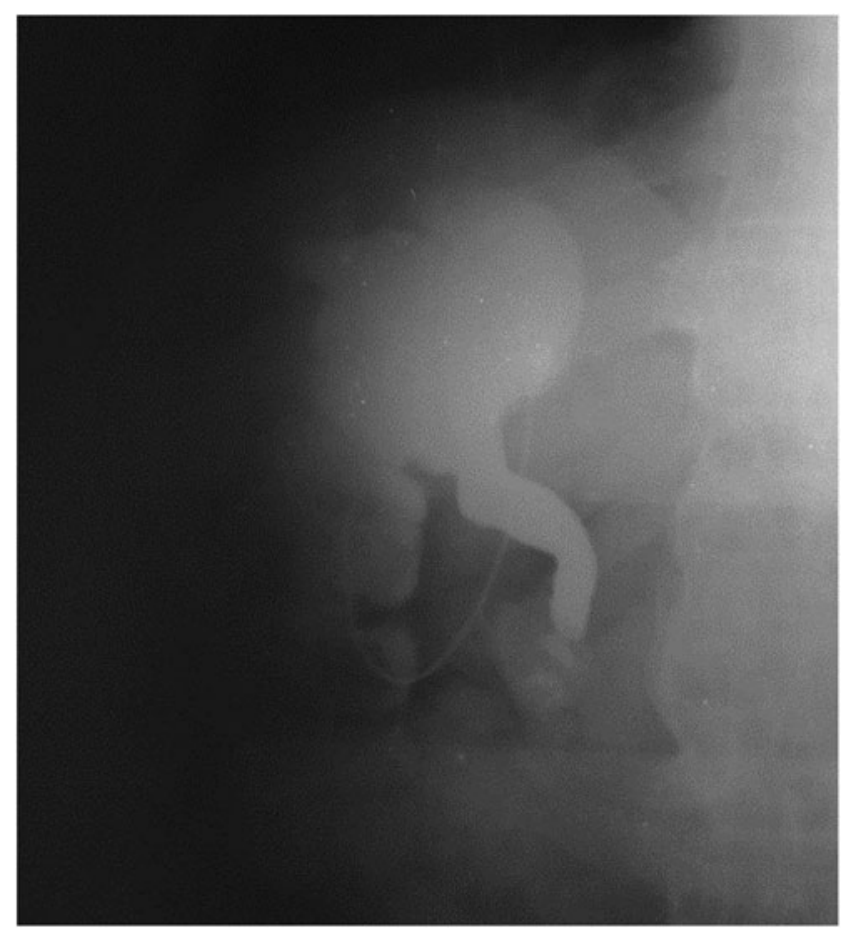

FIG. 2. Trans-cystic peri-operative cholangiography showing normal common bile duct with simultaneous opacification of hydatid liver cystic cavity and the right colonic angle. erating local ischemia infection, and necrosis contributes to gradual erosion of adjacent organs. This abnormal communication allows incomplete evacuation of hydatid cyst. Intra-hydatid cyst pressure will decrease, but infection and inflammatory lesions continue to develop. Clinical symptoms can include painful right hypochondriac region with fever, signs of main bile duct obstruction, and anaphylactic reaction. The presence of hydatid cysts in the stools (hydatidorrhea or hydatidenteria) [7] as well as membranes in the vomit (hydatid-emesis) in case of pulmonary hydatid cyst, can be found in such cases [3]. Diagnosis is generally performed by ultrasound and abdominal CT scan. This abnormal communication is always considerate as a result of hydatid cyst infection secondary to hydatid cyst-biliary fistula. A cholecystectomy associated to an intraoperative cholangiography are necessary. Intra-operative cholangiography can prove also this communication between cyst, colic, and biliary tract such as in our case. It can be done using trans-cystic, transvesicular, trans-fistula, or trans-choledochal approach drain. Treatment is usually difficult. It must be adapted to each type of lesion. Regarding the cyst with large biliary fistula, radical treatment offers good results but it is not always feasible. Their indications were limited essentially for cysts of the left hepatic lobe and the hepatic anterior border cyst [3]. Conservative treatment of complicated hydatid hepatic cysts is dominated by two methods: DITFO (internal trans-fistulary drainage) and resection of the protruding dome. Regarding the cysto-colonic fistula, performing a right colectomy is considered less risky than simple suture or colonic stoma. For these patients, the prognosis is not related to the hydatid cyst lesion but to the associated gastrointestinal lesions. The segment concerned, particularly the duodenum, may present problems of repair or exclusion. As for colonic and small bowel fistula, the evolution is generally suitable [7].

\section{Conclusion}

The rupture of a hydatid liver cyst in the gastrointestinal tract is rare and clinical diagnosis remains difficult. This abnormal communication is always secondary to hydatid cyst infection caused by hydatid cyst-biliary fistula. Ultrasound echography, abdominal CT scan, and intra-operative cholangiography can establish the diagnosis. Surgical treatment is sometimes difficult and prognosis depends on the gastrointestinal organ affected.

\section{Author Disclosure Statement}

Authors have no conflicts of interest or financial ties to disclose.

\section{References}

1. McManus DP, et al. Echinococcosis. Lancet 2003;362:12951304.

2. Kourias B. A propos de 2000 cas de kystes hydatiques operés, in Bréves considérations d'ordre statistique et chirurgical. 1961. p. 165-168.

3. Zaouche A. Liver hydatid surgery: About 2013 case. Tunis: Tunisian Association for Surgery, 1994.

4. Barabde M, et al. Hydatid cyst-colonic fistula: An exceptional complication. Int J Res Med Sci 2016;4:1260-1263.

5. Bougioukas IG, et al., Liver hydatid cyst perforated into the large bowel: A case report. Cases journal 2009;2:1. 
6. Lo Casto A, et al., Hydatid cyst of the liver communicating with the left colon. Br J Radiol 1997;70:650-651.

7. Noguera M, et al., Spontaneous duodenal fistula due to hepatic hydatid cyst. Abdominal Imaging 1993;18:234-236.

8. de Diego Choliz J, et al. Computed tomography in hepatic echinococcosis. Am J Roentgenol 1982;139:699-702.

Address correspondence to: Dr. Mohamed Ali Chaouch 28 Gafsa Street

Ksar Hellal

Monastir government, 5070

Tunisia

E-mail: Docmedalichaouch@gmail.com

\begin{tabular}{|c|}
\hline Abbreviations Used \\
$\mathrm{CT}=$ computed tomorgraphy
\end{tabular}

Cite this article as: Nacef $\mathrm{K}$, Chaouch MA, Khalifa MB, Chaouch A, Ghannouchi M, Maatouk M, Boudokhane M. Liver hydatid cyst complicated by biliary and colonic fistula diagnosed after pre-operative cholangiography. Surgical Infections Case Reports 2:1, 92-94, DOI: 10.1089/crsi.2017.0025 